\title{
Detection of the altered metabolites of Raji cells in the presence of Epstein- Barr virus (EBV) using 1HNMR Spectroscopy
}

\author{
Zahra Dindar $^{1}$, Reza Haj Hosseini ${ }^{2 *}$, Ruhollah Vahhabpoor ${ }^{3}$, Zahra Zamani ${ }^{4}$
}

1) Department of Biology, Faculty of Basic Science, Payame Noor University, Tehran, Iran

2) Department of Biology, Faculty of Science, Payame Noor University, Tehran, Iran

\author{
3) Medical Lab Technology Department, School of Allied Medical Sciences, Shahid Beheshti \\ University of Medical Sciences, Tehran, Iran.
}

4)Biochemistry Department,Pasteur Institute of Iran, Tehran, Iran.

*Corresponding Author:

Fax:, Tel:, E-mail: (hosseini@pnu.ac.ir), Postal address:

\begin{abstract}
EBV is one of the most common viruses in humans and is directly implicated in carcinogenesis. The present study aimed to detect the altered metabolites of Raji cells in the presence of Epstein-Barr virus (EBV) using 1H NMR by NOESY technique. The cells and EBV were maintained in RPMI 1640 and $1 \mathrm{ml}$ of cells were transfected into falcon tubes containing cells and were incubated at $37^{\circ} \mathrm{C}$ for 2 hours. Metabolites extracted using water or chloroform/methanol, lyophilized and sent for 1H NMR analysis by NOESY technique. The NMR spectrum comprising of Fourier Transformed information about metabolites in the control and treated were imported into MATLAB (v.7.8.0.347) software and the metabolic cycles were determined using Metabo-Analyst software. The data have demonstrated that infected cells leads to proliferation and subsequent immortalization of cell lines through cellular replication machinery recruitment and changes the metabolic profile and promotes vital metabolites such as the carbohydrates engage in pentose phosphate and glycolactic, biosynthesis of nucleotide and amino acid pathways. The results also indicate that essential amino acids are required for protecting viral structure and the function of viral genes. Therefore, EBV infection of cells leads to the sustained elevation of cell growth and cell immortalization.
\end{abstract}

Keywords:Raji cells, Epstein-Barr virus, 1H NMR Spectroscopy, Metabolites

\section{Introduction}


Epstein-Barr virus (EBV) is one of eight known human herpes virus types in the herpes family that was first isolated by Epstein in 1964 from cultured cells of Burkett's lymphoma frequently found in children of equatorial Africa[1,2]. It is a double-stranded DNA virus of about $170 \mathrm{~kb}$, and encodes about 80 genes. EBV is one of the most common viruses in humans and is directly implicated in carcinogenesis [3] which is associated with the nasopharynx[4], salivary gland [5], breast [6], bladder [7], kidney [8], uterine cervix [9], colon [10] and lung [11] cancers cell lines. EBV can cause infectious mononucleosis, also called mono, and other illnesses. EBV has coevolved and become ubiquitous in all human populations through its different hosts, its ability to establish lifelong latency, intermittent reactivation after primary infection and limited clinical symptoms in the majority of infected individual[12].

The Raji cell line of lymphoblast-like cells was established from a Burkitt's lymphoma of the left maxilla of an 11-year-old Negro male which have become invaluable tools for hematological research as they provide an unlimited amount of cellular material. Cells of the Raji line do not contain virus particles as demonstrated by electron microcopy and although the cells are resistant to vesicular stomatitis virus, this resistance is not transferred to other normally susceptible test cultures and an interferon-like inhibitor has not been found[13].

Metabolomics is broadly defined as the large-scale study of systematic identification and quantification of the small molecule metabolic products (the metabolome) of a biological system (cell, tissue, organ, biological fluid, or organism), which are the end products of cellular processes. Metabolome refers to the complete set of small-molecule metabolites (such as carbohydrate, amino acids, nucleotides, phospholipids, steroids, fatty acids, metabolic intermediates, hormones and other signaling molecules, and secondary metabolites)[14]. The metabolome forms a large network of metabolic reactions, where outputs from one enzymatic chemical reaction are inputs to other chemical reactions. Mass spectrometry and NMR spectroscopy are the techniques most often used for metabolomics profiling $[15,16]$.

Various studies have shown that EBV have been an important cause of cancer in human and is associated with a broad-spectrum of human cancers originating from epithelial cells, lymphocytes and mesenchymal cells. Recent advances in cancer research have demonstrated that Epstein Barr virus can alter the metabolites of infected cells and may result in the development of cancer in humans[4-11]. In recent years, LC-MS and 1H NMR have been established as the gold standard technique for metabolites analysis because of the technique's inherent analytical specificity and sensitivity[16,17]. The purpose of these efforts is for identification and quantification of metabolites that are uniquely correlated with an individual disease in order to accurately diagnose and treat the morbidity. Previous reports revealed that some metabolites (tripenoides) in raji cells may have inhibitory effects on the induction of Epstein-Barr virus early antigen (EBV-EA) by 12-O-tetradecanoylphorbol-13-acetate (TPA) which metabolites were determined on the basis of spectroscopic methods[18,19]. 
The purpose of precision medicine is to design disease prevention and treatment methods taking into account individual variability in environment, lifestyle, genetics, and molecular phenotype which metabolic phenotyping has the potential to generate high-volumes of complex spectral data[20]. The present study aimed to detect the altered metabolites of Raji cells in the presence of Epstein-Barr virus (EBV) by using 1H NMR Spectroscopy.

\section{Materials and methods}

\section{Cell line}

The Raji (B-cell lymphocyte) and EBV-producing marmoset B-cell (B95-8) cell lines were obtained from national Cell Bank of Iran (Pasteur Institute, Tehran, Iran)[21,22].

\section{EBV preparation}

B95-8 cells were cultivated in RPMI 1640 with $15 \%$ fetal bovine serum at $37^{\circ} \mathrm{C}$ and in $5 \% \mathrm{CO} 2$ humidified atmosphere. After 48 hours, the cells were centrifuged at $1300 \mathrm{rpm}$ for 4 min to separate EBV-containing culture supernatant from cells[22].

\section{Cell culture}

The Raji cells were maintained in RPMI 1640 supplemented with L-glutamine, 10\% FBS and $1 \%$ antibiotics (penicillin/streptomycin). The cells $\left(1 \times 10^{6}\right.$ cells $\left./ \mathrm{ml}\right)$ were plated in T-25 flasks containing $5 \mathrm{ml}$ of CGM and grown in a humidified incubator under an atmosphere of $95 \%$ air and $5 \% \mathrm{CO} 2$ at $37^{\circ} \mathrm{C}$ to sub confluence $(90-95 \%)$. The Raji cells were transferred to 6 falcon tubes and were centrifuged at $1300 \mathrm{rpm}$ for $5 \mathrm{~min}[23]$.

\section{Virus transfection}

Viruses were obtained from B95-8 cells. $1 \mathrm{ml}$ of cells had transfected into falcon tubes containing Raji cells and incubated at $37^{\circ} \mathrm{C}$ for 2 hours. After incubation, $4 \mathrm{ml}$ of CGM supplemented with $5 \%$ fetal bovine serum was added to each falcon tubes and the contents of the falcon tubes were transferred to a new flask. After one week, Raji cells were infected by EBV[23].

\section{PCR analysis}

Raji cells were seeded in dishes at 500,000 cells/10 mL/ $75 \mathrm{~cm} 2$. One day after seeding, the medium was changed, and the cells were incubated with the test compounds for $12 \mathrm{~h}$. At the end of the incubation, DNA was extracted using QIAGEN kit (QIA amp® DNA Mini \& Book Mini Handbook) and was refrigerated at $-20^{\circ} \mathrm{C}$. PCR for EBNA I gene was carried out using the specific primers which forward and reverse primers were prepared from LIGO company. The results of the PCR productshave been confirmed by an agarose gel electrophoresis. 
99

100

101

102

103

104

105

106

107

108

109

110

111

112

113

114

115

116

117

118

119

120

121

122

123

124

125

126

127

128

129

130

131

132

The method of extraction using methanol-chloroform-water was done as described previously. The temperature of the extraction procedure was maintained at $4{ }^{\circ} \mathrm{C}$ by working in a crushed ice bath. Cells were washed in $1 \mathrm{X}$ PBS and centrifuged at 6,000g for $5 \mathrm{~min}$ and resuspended in 500 $\mu \mathrm{L}$ of ice-cold $2: 1(\mathrm{v} / \mathrm{v})$ methanol-chloroform solution. It was then transferred into a $1.5 \mathrm{~mL}$ Eppendorf tube, $250 \mu \mathrm{L}$ of ice-cold $\mathrm{H} 2 \mathrm{O}$ 1:1 (v/v) chloroform/H20 was added and vortexed. The cells were sonicated on ice for $10 \mathrm{~min}$ and centrifuged for $5 \mathrm{~min}$ at $18000 \mathrm{Xg}$. The lower lipophilic and the upper hydrophilic extracts were separated and collected in different Eppendorf tubes and lyophilized and stored at $-20^{\circ} \mathrm{C}[24]$.

\section{H NMR preparation}

Lyophilized hydrophilic cell extracts $(n=10)$ were resuspended in $200 \mu \mathrm{L}$ of buffer $(150 \mathrm{mM}$ potassium phosphate at $\mathrm{pH} 7.4,1 \mathrm{mM} \mathrm{NaN3}$, and $0.01 \%$ trimethylsilyl propionate (TSP) (Sigma, CA, USA) in $100 \%$ D2O and the lipophilic cell extracts $(n=10)$ were resuspended in 200 $\mu$ Ldeuterated chloroform.

\section{H NMR spectroscopy}

The cell suspensions were placed in $5 \mathrm{~mm}$ probes for analysis and one dimensional spectroscopy was performed on a $1 \mathrm{H}$ NMR spectrometer (Bruker AV-500) with filed gradient operating at $500.13 \mathrm{MHZ}$ for observation of proton at $298 \mathrm{~K}$. One dimensional $1 \mathrm{H} \mathrm{NMR}$ spectra were acquired with $6009.6 \mathrm{~Hz}$ spectral width, a 10- $\mu$ s pulse $0.1 \mathrm{~s}$ mixing time, 3000 transients and 3.0 s relaxation delay, with standard 1D NOESY (nuclear Overhauser spectroscopy) pulse sequence to suppress the residual water peak. The 1H NMR spectrum comprising of Fourier Transformed information about metabolites in the control and treated groups(both hydrophilic and lipophilic extracts of each) were imported into MATLAB (v.7.8.0.347) software and first analyzed by ProMetab software (version 1.1) Chemical shifts between 0 and $10 \mathrm{ppm}$ were normalized and spectra binned in 0.004units and the water peak (4.7) removed. The Excel files were then assessed by PLS-Toolbox version 3.0 and Partial Least Square Discriminant Analysis (PLS-DA) was applied.

\section{Identification of metabolites}

The differentiating metabolites related to these resonances were identified by chemical shift determination using Human Metabolome Database Data Bank (HMDB) (http://www.hmdb. ca/metabolites). The metabolic cycles were determined using Metabo-Analyst software (http://www.metaboanalyst.ca/).

\section{Results}

Figure 1 shows Superimposed 1H NMR spectra of hydrophilic and lipophilic layers between experimental and control groups. 


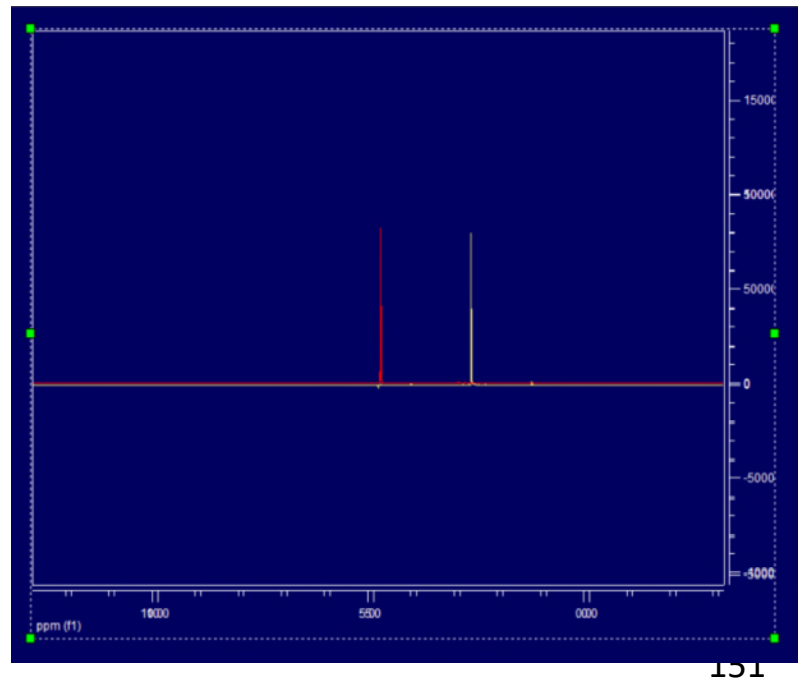

152

Figure A controls.

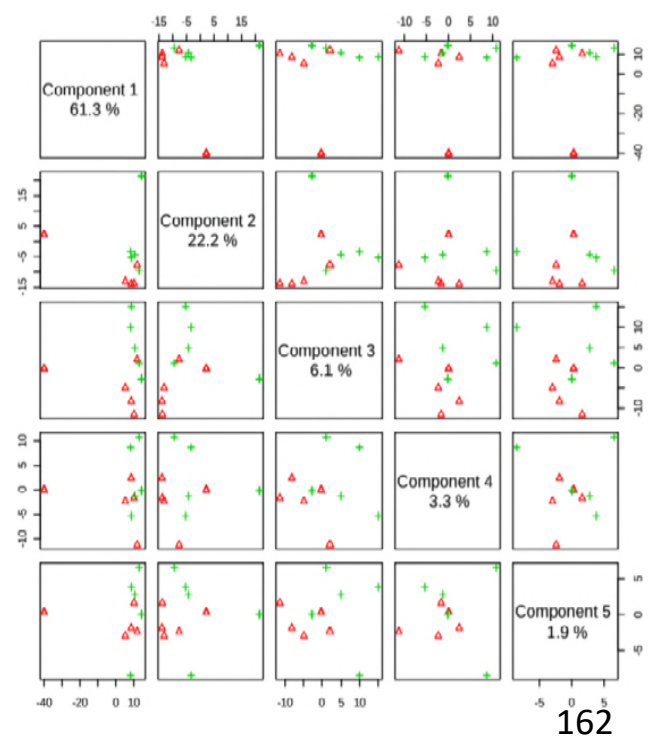

Figure AFigure B phase (B) using PLS-DA method.

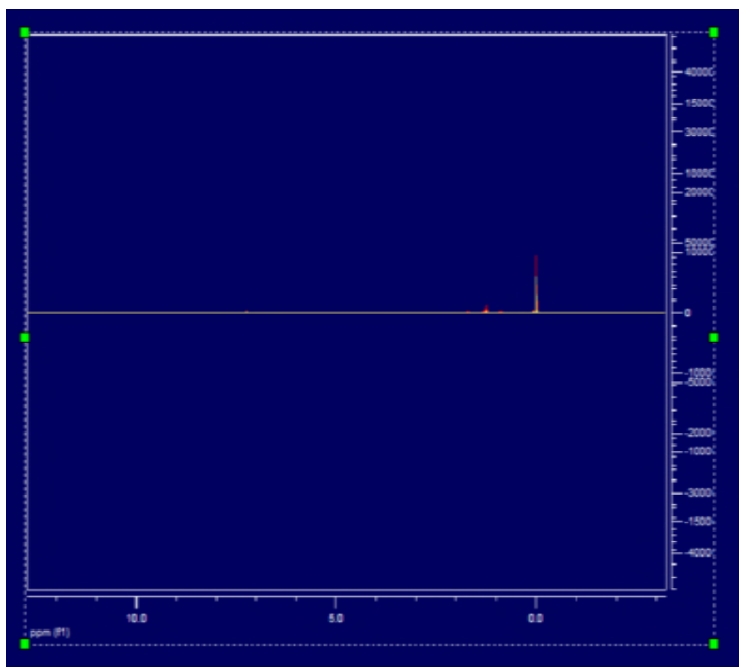

Figure B

Fig.1. Superimposed 1HNMR spectra ofhydrophilicphase (A) and lipophilic phase (B) of EBV treated Raji cells and

Figure 2 Indicates the analysis of $1 \mathrm{H}$ NMR spectra of hydrophilic and lipophilic layers is depicted as score plot using Partial Least Squares - Discriminant Analysis (PLS-DA) method.

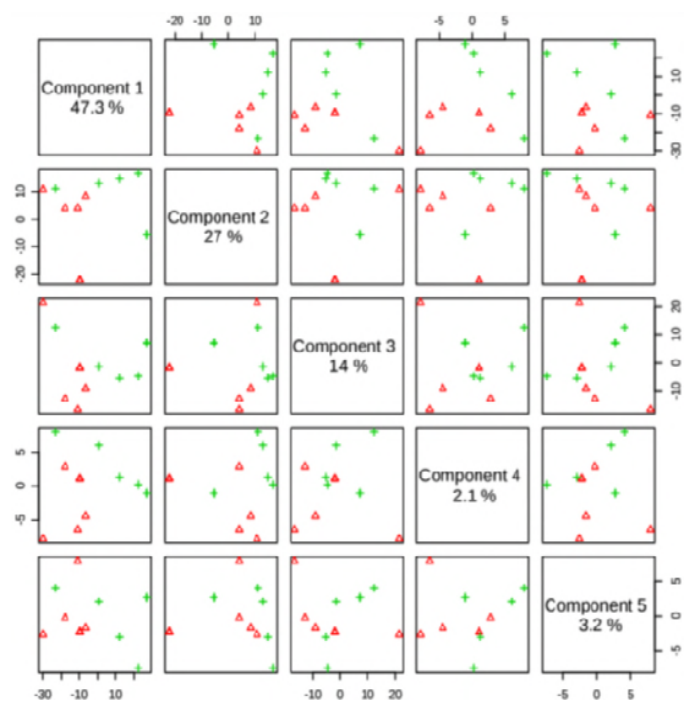

Fig.2. Score plot of Raji cells with EBV inexperimental and control groups inhydrophilic phase (A) and lipophilic 
Figure 3 shows loading plot of hydrophilic and lipophilic layers between experimental and control groups using PLS-DA method.
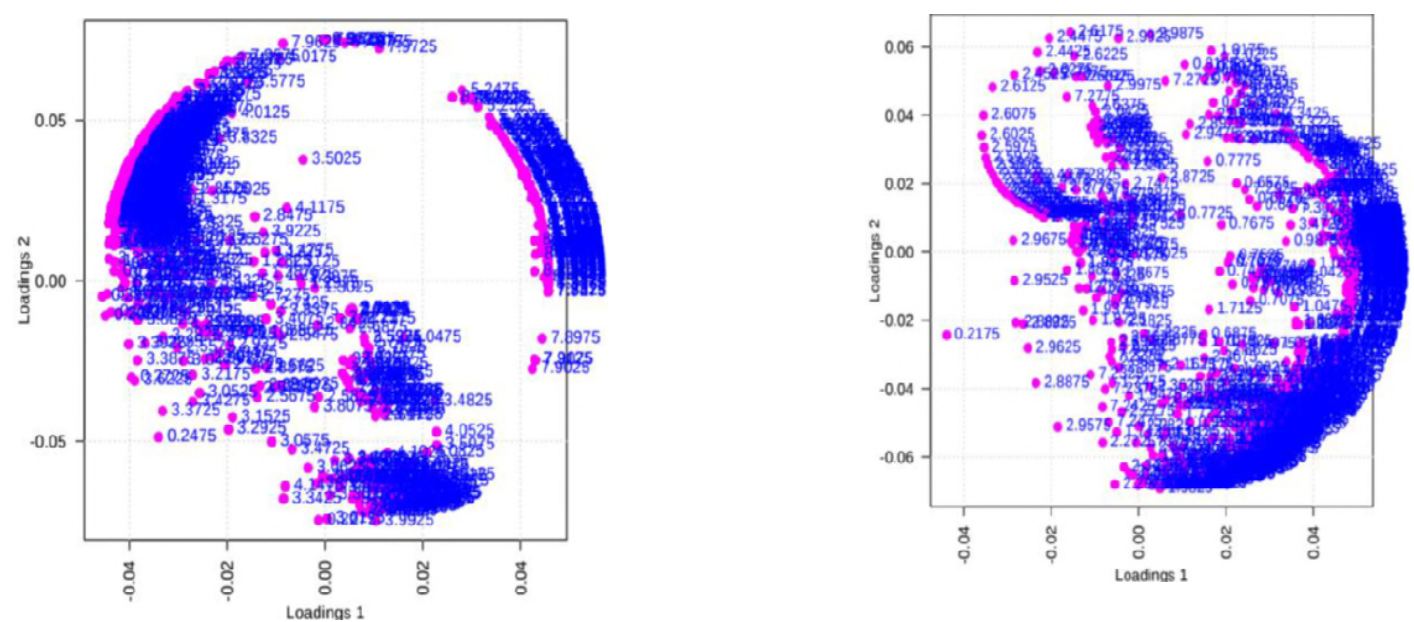

Figure A Figure B

Fig.3. Loading plot of Raji cells with EBV inexperimental and control groups inhydrophilic phase (A) and lipophilic phase (B) using PLA-DA method.

Figure 4 shows the affected metabolites of the raji cells in hydrophilic and lipophilic phase
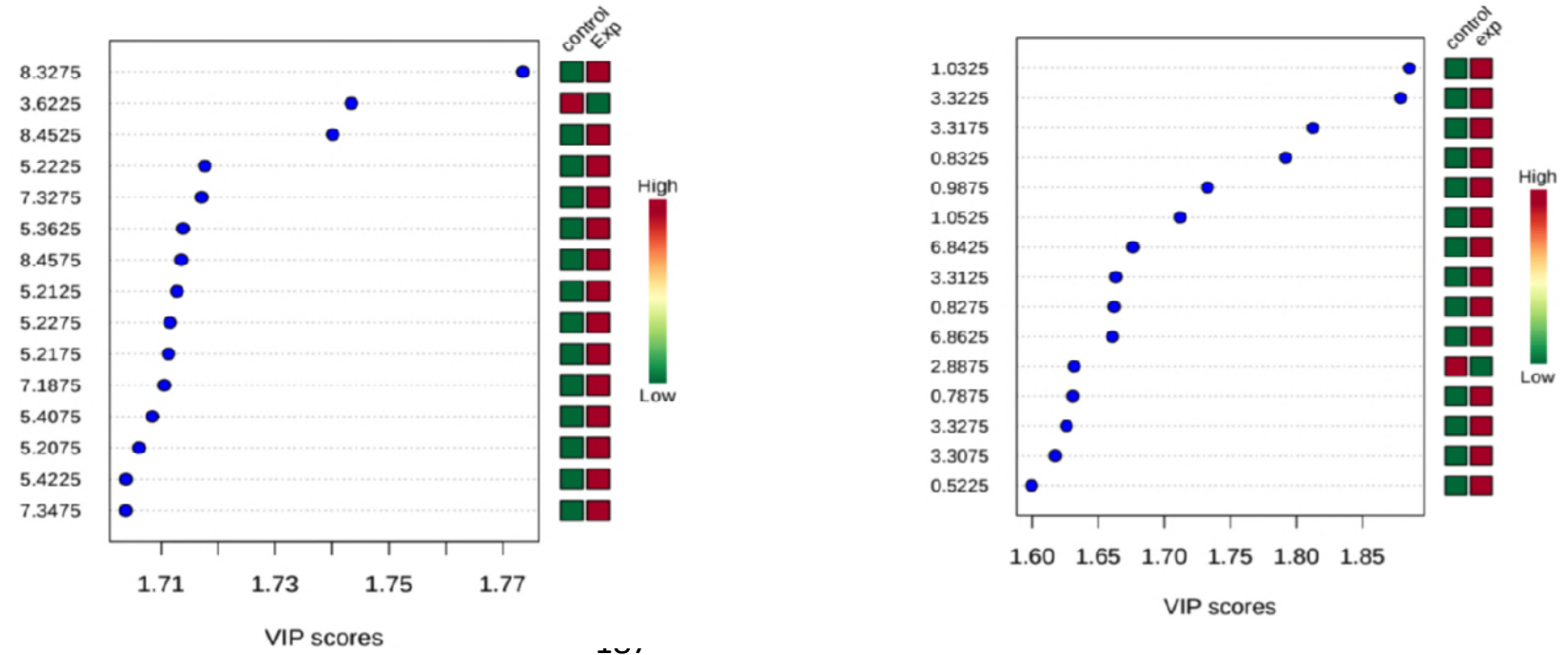

Figure AFigure B 
Table.1. Altered metabolites of treated group with EBV in hydrophilic phase on the base of HMDB.

Fig.4. Summary plot of over-representation analysis ofhydrophilic phase (A) and lipophilic phase (B) metabolites of Raji cells treated with EBV.

Table 1 showed the results of metabolites of Raji cells in hydrophilic phase treated by EBV which obtained from human metabolites data base (HMDB).

\begin{tabular}{|l|c|c|c|c|}
\hline \multicolumn{1}{|c|}{ Metabolite } & HMDB & $\begin{array}{c}\text { Chemical } \\
\text { Shift }\end{array}$ & levels & Abbreviation \\
\hline & & & & \\
L-Phenylalanine & HMDB00159 & 7.3475 & $\uparrow$ & L-Phe \\
Glucose 1-phosphate & HMDB01586 & 5.4225 & $\uparrow$ & Glu 1-P \\
L-Fucose & HMDB00174 & 5.2075 & $\uparrow$ & L-Fu \\
D-Ribose 5-phosphate & HMDB01548 & 5.4075 & $\uparrow$ & D-Rib \\
L-Tyrosine & HMDB00158 & 7.1875 & $\uparrow$ & L-Tyr \\
L-Fucose & HMDB00174 & 5.2175 & $\uparrow$ & L-Fuc \\
Glucose 6-phosphate & HMDB01401 & 5.2275 & $\uparrow$ & Glu 6-p \\
1-Methyladenosine & HMDB03331 & 5.2125 & $\uparrow$ & 1.Methyladenosine \\
PC(16:0/16:0) & HMDB00564 & 8.4575 & $\uparrow$ & PC \\
L-Phenylalanine & HMDB00159 & 5.3625 & $\uparrow$ & L- Phe \\
Glucose 6-phosphate & HMDB01401 & 7.3275 & $\uparrow$ & Glu 6-p \\
D-Ribose 5-phosphate & HMDB01548 & 5.2225 & $\uparrow$ & D-Rib 5-p \\
1-Methyladenosine & HMDB03331 & 8.4525 & $\uparrow$ & 1.Methyladenosine \\
Fructose 6-phosphate & HMDB00124 & 3.6225 & $\downarrow$ & Fru 6-p \\
Nicotinamideribotide & HMDB00229 & 8.3275 & $\uparrow$ & Nicotinamideribotide \\
& & & & \\
\hline
\end{tabular}
which obtained from human metabolites data base (HMDB) showed in Table 2. 
Table.2. Altered metabolites of treated group with EBV in lipophilic phase on the base of HMDB.

\begin{tabular}{|l|l|l|c|c|}
\hline \multicolumn{1}{|c|}{ Metabolite } & \multicolumn{1}{|c|}{ HMDB } & Chemical Shift & levels & Abbreviation \\
\hline & & & & \\
Cholesterol & HMDB00067 & 0.5225 & $\uparrow$ & Chol \\
L-proline & HMDB00162 & 3.3075 & $\uparrow$ & L-Pro \\
Beta-Leucine & HMDB03640 & 3.3275 & $\uparrow$ & B- Ala \\
Cholesterol & HMDB00067 & 0.7875 & $\uparrow$ & Chol \\
L-Arginine & HMDB0000168 & 2.8875 & $\downarrow$ & L-Arginine \\
5-Hydroxy-L-tryptophan & HMDB00472 & 6.8625 & $\uparrow$ & 5-Hydroxy-L-Try \\
Tryptophan & HMDB00929 & 6.8625 & $\uparrow$ & Try \\
Cholesterol sulfate & HMDB0000653 & 0.8275 & $\uparrow$ & Chol \\
L-Proline & HMDB00162 & 3.3125 & $\uparrow$ & L-Pro \\
S-Adenosylhomocysteine & HMDB00939 & 6.8425 & $\uparrow$ & SAH-Cys \\
2-Ketobutyric acid & HMDB00005 & 1.0525 & $\uparrow$ & 2- Ketobutyric acid \\
L-Valine & HMDB00883 & 0.9875 & $\uparrow$ & L-Val \\
Cholesterol & HMDB00067 & 0.8325 & $\uparrow$ & Chol \\
L-Proline & HMDB00162 & 3.3175 & $\uparrow$ & L-Pro \\
L-Proline & HMDB00162 & 3.3225 & $\uparrow$ & L-Pro \\
L-Valine & HMDB00883 & 1.0325 & & L-Val \\
& & & & \\
\hline
\end{tabular}

211

212

213

214

Figure 5 shows the affected metabolic pathways of the raji cells in hydrophilic phase treated by EBV using the pathway analyzing tool (MetaboAnalyst 3.0). 


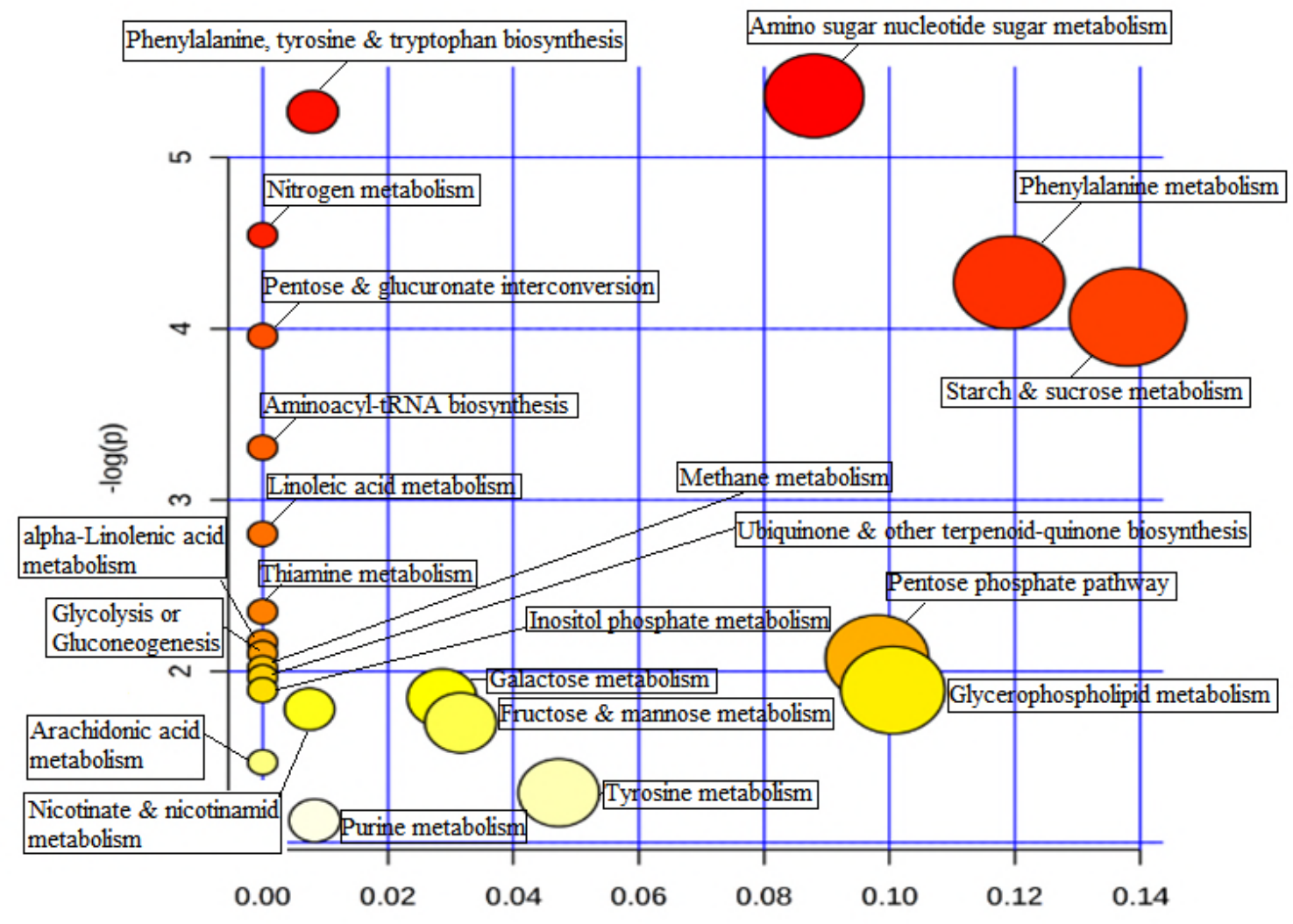

Pathway Impact

Fig.5.Important metabolicpathways in the hydrophilic of Raji cells treated with EBV using MetaboAnalyst 3.0.

Table 3 indicates the alteration of metabolic pathways of Raji cells in hydrophilic phase treated 


\begin{tabular}{|c|c|c|c|c|c|c|}
\hline Pathway & Total & Expected & Hits & Raw p & $-\log (p)$ & Metabolites \\
\hline $\begin{array}{l}\text { Amino sugar \& nucleotide sugar } \\
\text { metabolism }\end{array}$ & 88 & 0.37 & 3 & 4.70E-03 & $5.36 \mathrm{E}+00$ & Glu1p,Fru6p,Fuc \\
\hline $\begin{array}{l}\text { Phenylalanine,tyrosine\& } \\
\text { tryptophanbiosynthesis }\end{array}$ & 27 & 0.11 & 2 & $5.16 \mathrm{E}-03$ & $5.27 \mathrm{e}+00$ & Tyr, Phe \\
\hline Nitrogen metabolism & 39 & 0.16 & 2 & $1.06 \mathrm{E}-02$ & $4.55 \mathrm{E}+00$ & Tyr, Phe \\
\hline Phenylalanine metabolism & 45 & 0.19 & 2 & $1.40 \mathrm{E}-02$ & $4.27 \mathrm{E}+00$ & Tyr, Phe \\
\hline Starch and sucrose metabolism & 50 & 0.21 & 2 & $1.71 \mathrm{E}-02$ & 4.07E+00 & Glu1p,Glu 6p \\
\hline $\begin{array}{l}\text { Pentose and } \\
\text { glucuronateinterconversion }\end{array}$ & $\begin{array}{l}53 \\
75\end{array}$ & $\begin{array}{l}0.22 \\
0.31\end{array}$ & 2 & $\begin{array}{l}1.91 \mathrm{E}-02 \\
3.67 \mathrm{E} 02\end{array}$ & $\begin{array}{l}3.96 \mathrm{E}+00 \\
3.31 \mathrm{E}+00\end{array}$ & $\begin{array}{l}\text { Fru6p, Glu1p } \\
\text { Tyr, Phe }\end{array}$ \\
\hline Aminoacyl-tRNA biosynthesis & & & & & & \\
\hline
\end{tabular}

237 Figure 6 indicates the affected metabolic pathways of the raji cells in lipophilic phase treated by

238 EBV using the pathway analyzing tool (MetaboAnalyst 3.0). 


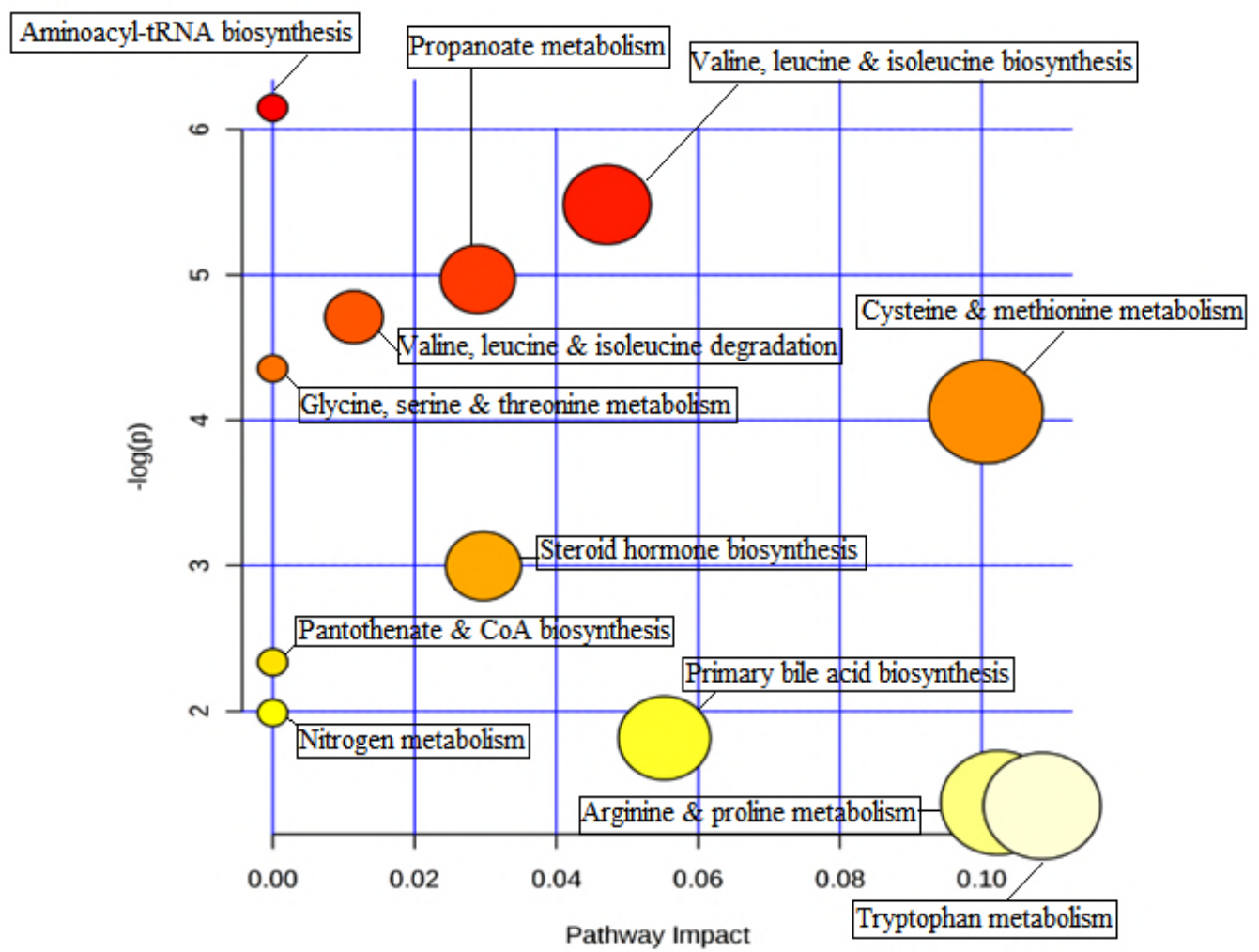

241 Fig.6.Important metabolicpathways in the lipophilic phaseofRaji cells treated with EBV using MetaboAnalyst 3.0.

243 Table 4 demonstrates the alteration of metabolic pathways of Raji cells in lipophilic phase 244 treated by EBV using the pathway analyzing tool (MetaboAnalyst 3.0). 
Table.4.

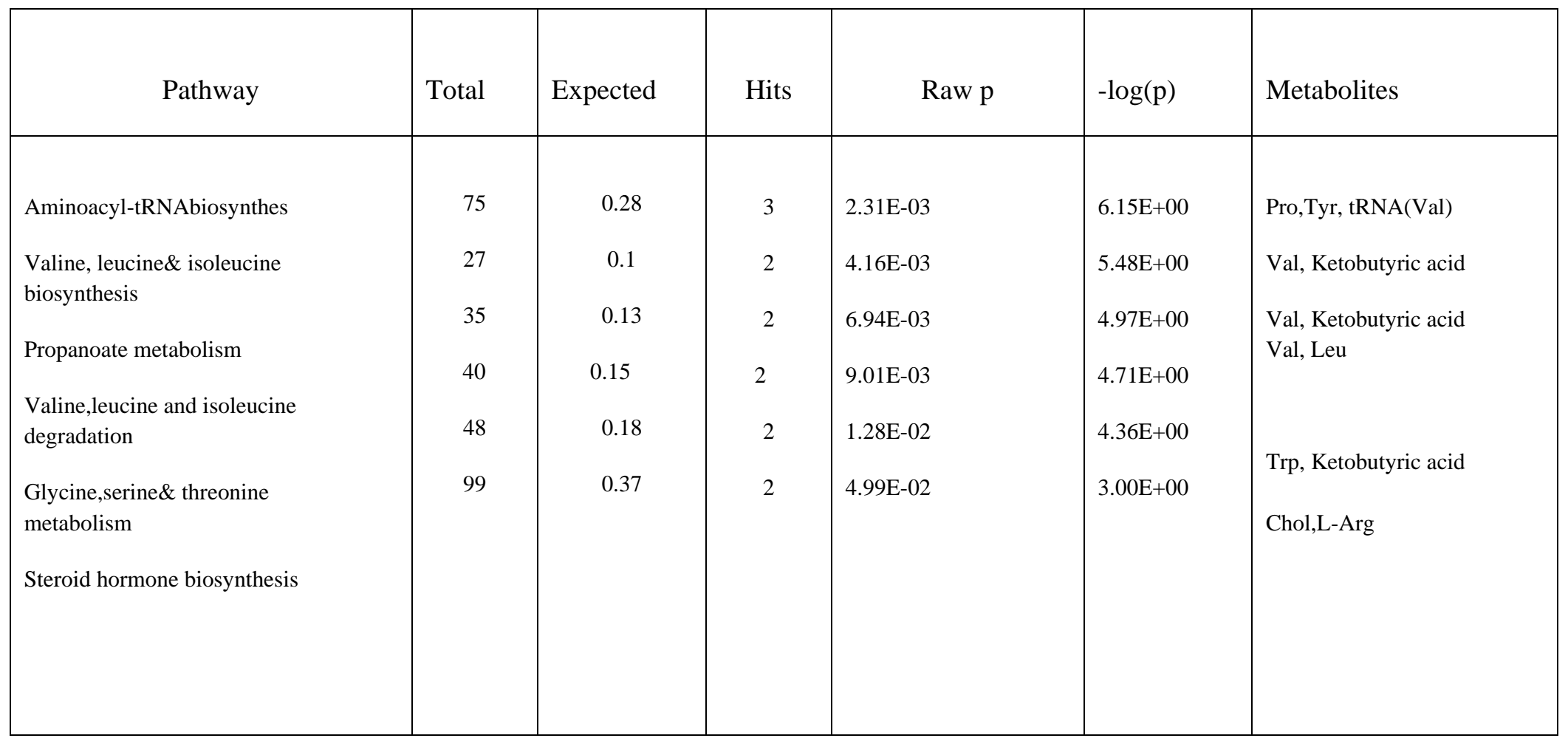

\section{Discussion}

258 A wide range of metabolites and chemical structure were measured upon extraction and samples

259 preparation, which berecognizedsignificantly, altered metabolic pathways according to

260 metaboAnalyst website.

261 According to the obtained results for hydrophilic phase, glucose 1-phosphate, glucose 6-

262 phosphate and fructose 6-phosphate have been the most frequency among metabolic pathways

263 including amino sugar and nucleotide sugar reaction, starch and sucrose metabolism and

264 pentose-glucuronateinterconversion. In lipophilic phase, proline, valine, tyrosine, triptophane,

265 and ketobutyric acid also have the most alteration among metabolic pathway including amino

266 acyl tRNA, valine, leucine and isoleucine biosynthesis, glycine, serine, threonine and propaonate

267 metabolism and valine, leucine and isoleucine degradation aromatic amino acids ( tyrosine and

268 phenylalanine ) also may be changed among tyrosine, phenylalanine, tryptophane and nitrogen

269 metabolic pathways. Although,the alteration in metabolic pathways have been resulted in

270 difference between Raji cells and EBV-infected cells.

271 The results indicated that amino sugar and nucleotide sugar metabolism are the most important

272 metabolic pathways in hydrophilic phase. The metabolites conversions, especially glucose 1- 
273 phosphate,fructose 6-phosphate and fucose were observed in these pathways, which they are the

274

275

276

277

278

279

280

281

282

283

284

285

286

287

288

289

290

291

292

293

294

295

296

297

298

299

300

301

302

303

304

305

306

307

308

309

310

311 most valuable metabolites in glycolysis pathway. The result from this study also support that changes in metabolite concentration, particularly rising glucose and L-fucose levels were found in EBV-infected raji cells in comparison with control cells. It also hasbeenshown that fructose 6phosphate and glucose 1-phosphateare involved in pentose-glucuronateinterconversion pathway. In line with this investigation, some virological studies showed that EBV lead to change in metabolic assay from early infection to long-term outgrowth that may be stimulate glucose import and surface glucose transporter-1 (Glut-1) levels, result in induction of glycolysis, oxidative phosphorylation and suppression of basal autophagy[25]. There are some reports showing that serum glycoprotein L-fucose levels have two-fold rise in head and neck neoplasma compared to control group[26]. Advanced in cancer have been shown that fucose may be is useful in breast cancer treatments and $\alpha$-L-fucose has pivotal role in construction of malignant and metastaric phenotype of various human breast cancer cell lines. Additionally, some breast cancer cell lines biomarkers are fucose-rich[27].

Metabolomics approach demonstrate alteration in glycolysis metabolites is associated with EBV in nasopharyngeal carcinoma (NPC) which overexpression of EBV-encoded latent protein 1 (LMP1) may lead to glycolysis induction. Some glycolysis genes (i.e. hexokinase 2) have central roles in LMP1-mediated glucose metabolism reprogramming in NPC cells. Additionally, positive correlation was existed between HK2 and LMP1 in NPC biopsies, and the HK2 induction was associated with poor survival of NPC patient after radiation therapies[28]. Therefore, there is a potential correlation between glycolysis pathway and EBV infected cells. Furthermore, the pentos phosphate pathway is required for ribonocleotide synthesis and NADPH production which branches from glycolysis [29].At first this research suggests phenylalanine and tyrosine are involved in nitrogen metabolism pathway. According to virologycal studies, two phenylalanine (F600, F605) are located in R transactivator (Rta) c-terminal which play pivotal role in DNA binding to target cells. These two phenylalanine are essential for Rta expression which Rta activates the EBV lytic cycle. If two other aromatic amino acids (Tryptophan and Tyrosine) are substituted with two phenylalanine, maintenance of mRNA activity of the BMLF1 gene have been seen. However, substitution of Tryptophan and Tyrosine with non-aromatic amino acid including Alanine and Valine, lead to capacity elimination of Rta activity. Valine and glycine substitution instead of phenylalanine in Rta protein act as inhibitor and may prevent its DNA-binding function. The EBV BZLF1 protein (ZEBRA, Zta) aromatic amino acids including (phenylalanine, Tyrosine and Tryptophan) are crucial components of activation domain,thereforeRta play important role in EBV lytic cycle by the subsitution of cellular signaling pathway and synergy with EBV ZEBRA protein [30].

Moreover, the results in lipophilic phase revealed that proline, valine, tyrosine, tryptophane amino acids and ketobutyric acid are altered in metabolic pathway of tRNA, valine, leucine, isoleucine, serine, glycine, threonine and propanoate biosynthesis, and degradation of valine, leucine and isoleucine. Amino acyletRNA biosynthesis could be among the first pathways in 
312 lipophilic phase which several amino acids such as proline, tyrosine and valine can be altered in

313 thesepathways. N-terminal domain of LMP2A contains eight phosphorylated tyrosine

314 residue.Two of which constitute an immunoreceptor tyrosine activation motif (ITAM)[31].

315 ITAM consist of a pared tyrosine and leucine residues and play a pivotal role in signal

316 transduction of $\mathrm{B}$-cell receptors $(\mathrm{BCR})$ and $\mathrm{T}$-cell receptors, lymphocyte proliferation and

317 activity of kinase families. Although, LMP2A ITAM motif participate in BCR signal

318 transduction as an inhibitor[32]. Several lines of studies suggest that LMP2A prevents BCR

319 signal transduction trough engaging Nedd-4 ubiquitin protein ligases to promote the degradation

320 of Lyne and LMP2A by an ubiquitin- dependent mechanism[33].

321 The results showed in lipophilic phase also indicating that valine and acid butyric are involved

322 and altered in metabolic pathway of valine, leucine and isoleucine biosynthesis. Several

323 investigation consider that the $\mathrm{C}$-myc transcription factor is acting as a proto-oncogene which

324 activation of MYC lead to promotion of cell cycle transition and is recognized as a leucine zipper

325 protein which is activation by mitogenic factors under normal circumstances[24]. On this base,

326 previous studies on EBV genome have shown that multi-nucleonal proteins have a highly

327 charged $\mathrm{N}$-terminus which may provide nuclear signals and contains heptad repeats of leucine,

328 isoleucine or valine that can act as dimerization domain; the third exon includes leucine and

329 isoleucine heptad repeats which make possible intraction of coiled-coild and facilities in

330 hemodimerization of BZLF1[35].

331 In present study tryptophane level has dramatically increased in glycine, serine and threonine

332 metabolic pathways. Valine and ketobutyric acid also have seen to be engaged in propaonate

333 metabolism'srecent data, indicating that Tryptophane is essential for virus penetration in cells

334 and crosses lipid bilayers without pore formation[36].

335 In steroid hormone metabolism, the most alteration was observed in amount of cholesterol

336 metabolites. Some investigation has revealed that cholesterol enriched- domains in plasma membranes may are required for infection of human B-cells with EBV and is necessary for

338 membrane fusion, receptor localization in membrane micro-domain and early viral signaling events. Lipid rafts are also involved in MHC class II protein function[37].

Additionally, the finding results demonstrate that L-Argenine level has significantly reduced in cells exposed to EBV compared to control cells. There is various reports indicating L-Argenine supplementation may be lead to inhibition of spontaneous EBV reactivation in another Burkitts lymphoma cell line EB1 and B lymphoblastic cell line OB. L-Argenine also can induces inducible NO synthase and generates NO, which inhibits EBV reactivation in EBV-possitive cells[38].

The conclusionof this research showed that infection of Rajicells with EBV leads to proliferation and subsequent immortalization of cell lines through cellular replication machinery recruitment 
glucose-1-phosphate and fructose 1-phosphate) engage in pentose phosphate and glycolactic, biosynthesis of nucleotide and amino acid pathways. Theresults also indicate that essential amino acids are required for protecting viral structure and the function of viral genes. Furthermore, rates of proteins synthesis and function of glycolysis pathway give rise to increase in EBV-infected Raji cells compare with control cells. Therefore, EBV infection of Raji cells leads to the sustained elevation of cell growth and cell immortalization.

\section{Acknowledgement}

I would like to express my thanks to Pasteur Institute of Iran's Research council members especially Dr. M.R Aghasadeghi as the head of Hepatitis \& AIDS \& also staf of Pasteur Institute of Iran. Finally, I would like to express my appreciation to Dr. Hajhosseini as the head of Payam Noor University of Tehran for his supports.

\section{Conflict of interest statement}

The authors state that there are no conflicts of interest regarding the publication of this article.

\section{References}

1. M. Rowe, L. Fitzsimmons,A.IBell.Epstein-Barr virus and Burkittlymphoma.Chin J Cancer. 33 (2014) 609-619.

2. M.P. Thompson, R. Kurzrock. Epstein - Barr virus and Cancer.10 (2004) 803-821.

3.Y.H Ko. EBV and human cancer. ExpMol Med. 47(2015) 130.

4. J.K.C. Chan, F.Bray, P. McCarron, W. Foo, A.W.M. Lee, T. Yip. Nasopharyngeal carcinoma. In: Barnes L., Eveson J.W., Reichart P., Sidransky D., editors. WHO classification of tumors, pathology and genetics of head and neck tumours. IARC; Lyon (2005) 85-97.

5.S.J. Hamilton-Dutoit, M. Hamilton-Therkildsen, N.H. Nielsen, H. Jensen, J.P.H. Hensen, G. Pallesen Undifferentiated carcinoma of the salivary gland in Greenland Eskimos: demonstration of Epstein-Barr virus DNA by in situ hybridization. Hum Pathol. 22(1991) 811-815.

6. L.G. Labrecque, D.M. Barnes, I.S. Fentiman, B.E. Griffin. Epstein-Barr virus in epithelial cell tumors: a breast carcinoma study. Carcinoma Res. 55 (2001) 39-45.

7. P. Gazzaniga, R. Vercillo, A. Gradilone, I. Silvestri, O. Gandini, M. Napolitano. Prevalence of papillomavirus, Epstein-Barr virus, cytomegalovirus, and herpes simplex virus type 2 in urinary bladder cancer. J Med Virol. 55 (1998) 262-267.

8. M. Shimakage, K. Kawahara, S. Harada, T. Sasagawa, T. Shinka, T. Oka. Expression of Epstein-Barr virus in renal cell carcinoma. Oncol Rep. 18 (2007) 41-46.

9. T. Sasagawa, M. Shimakage, M. Nakamura., J. Sakaike, H. Ishikawa, M. Inoue. Epstein-Barr virus (EBV) genes expression in cervical intraepithelial neoplasia and invasive cervical cancer: a comparative study with human papillomavirus (HPV) infection. Hum Pathol. 31 (2000) 318-326. 
10. H.X. Liu, Y.Q. Ding, Y.O. Sun, L. Liang, Y.F. Yang, Z.L. Qi. Detection of Epstein-Barr virus in human colorectal cancer by in situ hybridazition. Di Yi Jun Yi Da XueXueBao. 22 (2002) 915-917.

11. C.Y. Castro, M.L. Ostrowski, R. Barrios, L.K. Green, H.H. Popper, S. Powell. Relationship between EpsteinBarr virus and lymphoepithelioma-like carcinoma of the lung: a clinicopathologic study of 6 cases and review of the literature. Hum Pathol. 32 (2001) 863-872.

12. D.J. McGeoch, S. Cook, A. Dolan, F.E. Jamieson, E.A. Telford. Molecular phylogeny and evolutionary timescale for the family of mammalian herpesviruses. J. Mol. Biol. 247(1995)443-458.

13. M.B. Karpora,J. Schoumans,I. Ernberg, J.I. Henter, M. Nordenskjold, B. Fadeel. Raji revisited: Cytogenetics of the original Burkitt's Lymphoma cell line. Leukemia. 19 (2005)159-161.

14. W. Weckwerth. Metabolomics in systems biology. Annu Rev Plant Biol. 54 (2003) 669-89.

15. A. Alonso,S. Marsal, A. Julià. Analytical Methods in Untargeted Metabolomics: State of the Art in 2015.Front BioengBiotechnol. 3 (2015) 23.

16. Z. Lei, DV. Huhman, L.W. Sumner. Mass Spectrometry Strategies in Metabolomics.JBiol Chem. 286(2011) 25435-25442.

17. J.L. Wagstaff, R.J. Masterton, J.F. Povey, C.M. Smales, M.J. Howard. 1H NMR Spectroscopy Profiling of Metabolic Reprogramming of Chinese Hamster Ovary Cells upon a Temperature Shift during Culture.PLoS One. 2013; 8(10): e77195.

18. G.J. Kapadia, M.A. Azuine, J. Takayasu, T. Konoshima, M. Takasaki , H. Nishino. Inhibition of epstein-barr virus early antigen activation promoted by 12-O-tetradecanoylphorbol-13-acetate by the non-steroidal antiinflammatory drugs. Cancer Lett. 161(2000)221-9.

19. M. Ukiya, T. Akihisa, H. Tokuda, M. Toriumi, T. Mukainaka, N. Banno. Inhibitory effects of cucurbitane glycosides and other triterpenoids from the fruit of Momordicagrosvenori on epstein-barr virus early antigen induced by tumor promoter 12-O-tetradecanoylphorbol-13-acetate. J Agric Food Chem. 50(2002)6710-5.

20.H. Hampel, S.E. O’Bryant, J.I. Castrillo, C. Ritchie, K. Rojkova, K. Broich. PRECISION MEDICINE - The Golden Gate for Detection, Treatment and Prevention of Alzheimer's Disease. J PrevAlzheimers Dis. 3(2016) 243259.

21. B.E. García-Pérez, J.J. De la Cruz-López, J.I Castañeda-Sánchez, A.R. Muñóz-Duarte, A.D. Hernández-Pérez, H. Villegas-Castrejón. Macropinocytosis is responsible for the uptake of pathogenic and non-pathogenic mycobacteria by B lymphocytes (Raji cells). BMC Microbiol.12 (2012) 246.

22. M. Bernasconi, C. Berger, J. Asigrist, A. Bonanomi, J. Sobek, F. Niggle, D. Nadal. Quantitative Profiling of housekeeping \& Epstein- Barr virus gene transcription in Burkitt lymphoma cell lines using an oligonucleotide microarray.Virol J. 3 (2006) 43.

23. F. Regina,N. Bernhard, B. Helmut, G. Karsten, S.L. Claire, D. Henri- Jacques. Epstein- Barr Virus B95.8 produced in 293 cells shows marked tropism for differentiated primary epithelial cells \& reveals interindividual variation in susceptibility to viral infection. IJC International Journal of Cancer. 12(2007) 588-594. 
24. N. Parvizzadeh, S. Sadeghi, S. Irani, A. Iravani, Z. Kalayee, N.A. Rahimi, M. Azadi,Z. Zamani. A Metabonomic Study of the Effect of Methanol Extract of Ginger on Raji Cells Using (1)HNMR Spectroscopy. Biotechnol Res Int. 57 (2014) 25-34.

25. K. McFadden, A.Y. Hafez, R. Kishton, J.E. Messinger, P.A. Nikitin, J.C. Rathmell, M.A. Luftig. Metabolic stress is a barrier to Epstein Barr virus- mediated B- cell immortalization.ProcNatlAcad U.S.A. 113(2016)782-790.

26. K.S.S. Rathan, K.B. Satheesh,K. Arunava. Significance of Serum L-Fucose Glycoprotein as Cancer Biomarker in Head and Neck Malignancies without Distant Metastasis. J clinDiagn Res. 7(2013)2818-2820.

27. J.J. Listinsky, G.P. Siegal, C.M. Listinsky. The emerging importance of $\alpha$ L-Fucose in human breast cancer. A review. AmJ Trans. 3(2011)292-322.

28. L. Xiao, Z.Y. Hu, X. Dong, Z. Tan, W. Li, M. Tang. Targeting Epstein-Barr virus oncoprotein LMP1-mediated glycolysis sensitize nasopharyngeal carcinoma to radiation therapy. Oncogen. 33 (2014) 4568-4578.

29. C.P. Krushna, H. Nissim. The Pentose phosphate pathway and cancer.Trends in Biochemical Sciences. 39(2014) 347-354.

30. L.W. Chen, V. Raghavan, P.J. Chang, D. Shedd, L. Heston, H.J. Delecluse. Two phenylalanines in the Cterminus of Epstein-Barr virus Rta protein reciprocally modulate its DNA binding and transactivation function. Virology.386(2009)448-61.

31. M. Fukuda, Y. Kawaguchi. Role of the Immunoreceptor Tyrosine-Based Activation Motif of Latent Membrane Protein 2A (LMP2A) in Epstein-Barr Virus LMP2A-Induced Cell Transformation.J Virol. 88(2014) 5189-5194.

32. S. Fruehling, R. Longnecker. The immunoreceptor tyrosine-based activation motif of Epstein-Barr virus LMP2A is essential for blocking BCR-mediated signal transduction. Virology. 235(1997)241-51.

33. M.A. Morris, C.W. Dawson, L.S. Young. Role of the Epstein-Barr virus-encoded latent membrane protein-1, LMP1, in the pathogenesis of nasopharyngeal carcinoma. Future Oncol. 5(2009)811-25.

34. C.V. Dang. MYC on the path to cancer. Cell. 149(212)22-35.

35. P. Tornero, E. Mayda, M.D.Gómez, L. Cañas, V. Conejero, P. Vera. Characterization of LRP, a leucine-rich repeat (LRR) protein from tomato plants that is processed during pathogenesis. Plant J.10(1996)315-30.

36. S.P. Soni, E. Adu-Gyamfi, S.S. Yong, C.S. Jee, R.V. Stahelin. The Ebola virus matrix protein deeply penetrates the plasma membrane: an important step in viral egress. Biophys J. 104(2013)1940-9.

37. R.B Katzman, R. Longnecker. Cholesterol-dependent infection of Burkitt's lymphoma cell lines by Epstein-Barr virus. J Gen Virol. 84(2003)2987-92.

38. H. Agawa, K. Ikuta, Y. Minamiyama, M. Inoue, T. Sairenji. Down-regulation of spontaneous Epstein-Barr virus reactivation in the P3HR-1 cell line by L-arginine. Virology. 304(2002)114-24. 